\title{
Last Borexino Result
}

\author{
Marcin Misiaszek* \\ Marian Smoluchowski Institute of Physics \\ Jagiellonian University \\ ul. Reymonta 4, 30-059 Krakow, Poland \\ E-mail: marcin.misiaszek@uj.edu.pl
}

On Behalf of the Borexino Collaboration:

C. Arpesella, H. O. Back, M. Balata, G. Bellini, J. Benziger, S. Bonetti, A. Brigatti, B.

Caccianiga, L. Cadonati, F. Calaprice, C. Carraro, G. Cecchet, A. Chavarria, M. Chen, F.

Dalnoki-Veress, D. DÁngelo, A. de Bari, A. de Bellefon, H. de Kerret, A. Derbin, M. Deutsch, A. di Credico, G. di Pietro, R. Eisenstein, F. Elisei, A. Etenko, R. Fernholz, K. Fomenko, R. Ford, D. Franco, B. Freudiger, C. Galbiati, F. Gatti, S. Gazzana, M. Giammarchi, D. Giugni, M. Goeger-Neff, T. Goldbrunner, A. Goretti, C. Grieb, C. Hagner, W. Hampel, E. Harding, S. Hardy, F. X. Hartman, T. Hertrich, G. Heusser, Aldo Ianni, Andrea Ianni, M. Joyce, J. Kiko, T. Kirsten, V. Kobychev, G. Korga, G. Korschinek, D. Kryn, V. Lagomarsino, P. Lamarche, M. Laubenstein, C. Lendvai, M. Leung, T. Lewke, E. Litvinovich, B. Loer, P. Lombardi, L. Ludhova, I. Machulin, S. Malvezzi, S. Manecki, J. Maneira, W. Maneschg, I. Manno, D. Manuzio, G. Manuzio, A. Martemianov, F. Masetti, U. Mazzucato, K. McCarty, D. McKinsey, Q. Meindl, E. Meroni, L. Miramonti, D. Montanari, M. E. Monzani, V. Muratova, P. Musico, H. Neder, A. Nelson, L. Niedermeier, L. Oberauer, M. Obolensky, M. Orsini, F. Ortica, M. Pallavicini, L. Papp, S. Parmeggiano, L. Perasso, A. Pocar, R. S. Raghavan, G. Ranucci, W. Rau, A. Razeto, E. Resconi, P. Risso, A. Romani, D. Rountree, A. Sabelnikov, R. Saldanha, C. Salvo, D. Schimizzi, S. SchÃünert, T. Shutt, H. Simgen, M. Skorokhvatov, O. Smirnov, A. Sonnenschein, A. Sotnikov, S. Sukhotin, Y. Suvorov, R. Tartaglia, G. Testera, D. Vignaud, S. Vitale, R. B. Vogelaar, F. von Feilitzsch, R. von Hentig, T. von Hentig, M. Wojcik, M. Wurm, O. Zaimidoroga, S. Zavatarelli and G. Zuzel

Borexino is a large-volume liquid scintillator detector placed in the underground halls of the Laboratori Nazionali del Gran Sasso in Italy. It is able to detect in real time neutrino interactions below $2 \mathrm{MeV}$, due to the very high radio-purity reached by the detector. The interaction rate of the $0.862 \mathrm{MeV}^{7} \mathrm{Be}$ neutrinos is $49 \pm 3$ (stat) \pm 4 (syst) counts/(day. 100 ton), in agreement with the oscillation hypothesis in the MSW Large Mixing Angle scenario. Our result is the first direct measurement of the survival probability for solar $v_{e}$ in the transition region between matter-enhanced and vacuum-driven oscillations.

European Physical Society Europhysics Conference on High Energy Physics, EPS-HEP 2009,

July 16 - 222009

Krakow, Poland

* Speaker. 


\section{Introduction}

Borexino [1] is a real-time experiment for low energy neutrino spectroscopy, operating since May 16th 2007 at the Laboratori Nazionali del Gran Sasso (Italy). Borexino is the first experiment to report a real-time observation of low energy solar neutrinos below 4.5 MeV [2], which were not accessible so far with the state-of-the art detector technologies because of natural radioactivity.

The results reported in this work present the real-time measurement of the low energy $(0.862$ $\mathrm{MeV}){ }^{7} \mathrm{Be}$ solar neutrinos with the Borexino detector from an analysis of 192 live days in the period from May 16, 2007 to April 12, 2008, totaling a 41.3 ton-yr fiducial exposure to solar neutrinos. The neutrino oscillations were first discovered in experiment which detected high energy cosmic radiation. Many years before solar neutrino experiments presented the lack of electron neutrinos. Elements of PMNS matrix are deduced mutually from low and high energy neutrino experiments. The real time measurement of the flux of sub $\mathrm{MeV}$ solar neutrinos is presently very precious to confirm the low energy behavior of the neutrino survival probability Pee predicted by the LMAMSW scenario: in fact neutrino oscillations are expected to be dominated by matter effects for energies higher than about $3 \mathrm{MeV}$ and by vacuum effects below $0.5 \mathrm{MeV}$ [3] . Therefore the first simultaneous measurement of the neutrino survival probability $P_{e e}$ out of both, vacuum and matter enhanced oscillation regions, can be announced. An additional physics result already obtained by Borexino concerns best limits for CNO flux, $v$ magnetic moment and Pauli principle violation.

\section{The Borexino Detector}

Borexino is a unsegmented calorimeter with on a active mass of 278 tons of organic liquid scintillator (Pseudocumene - 1,2,4 trimethylbenzene- doped with $1.5 \mathrm{~g} / \mathrm{l}$ of PPO -2,5 diphenyloxazole) contained in a thin $(125 \mu \mathrm{m})$ nylon vessel having 8.5 meter diameter. Solar neutrinos are detected by their elastic scattering on the electrons of the scintillators. A stainless steel sphere (13.7 $\mathrm{m}$ diameter), surrounding the scintillator vessel, houses the 2212 photomultipliers that detect the scintillation light. The volume between the two spheres is filled again by Pseudocumene but with the addition of a light quencher. A cylinder of $18 \mathrm{~m}$ diameter and $16.9 \mathrm{~m}$ height filled by ultrapure water contains the sphere and act both as radiation shield and as Cerenkov detector used to identify (and veto) muons. Key features of the scintillator are the high light yield (500 detected phe/Mev), the fast time response that allows to reconstruct the scintillation position and it permits the software definition of a fiducial volume, the possibility to discriminate between $\alpha$ and $\beta$ induced scintillations. The isotropy of the light emission forbids the reconstruction of the neutrino arrival direction which is a powerful tool to discriminate signals from background in water Cerenkov detector. The shape of the energy spectrum of solar neutrinos is the only strong signature available in the data: events induced by $\gamma$ or $\beta$ radioactivity cannot be distinguished from the signal on a event by event analysis but only through their spectral shape. This fact, together with the expected low signal rate (from few tens event/(days $100 \mathrm{t}$ ) to less than one event/(days $100 \mathrm{t}$ ) depending on the solar neutrino spectral component) strongly demands for an extremely high radiopurity detector. Borexino has reached, and in some case also exceeded, the design requirements about radiopurity. This is obtained using the principle of graded shielding, with the inner core scintillator at the center 

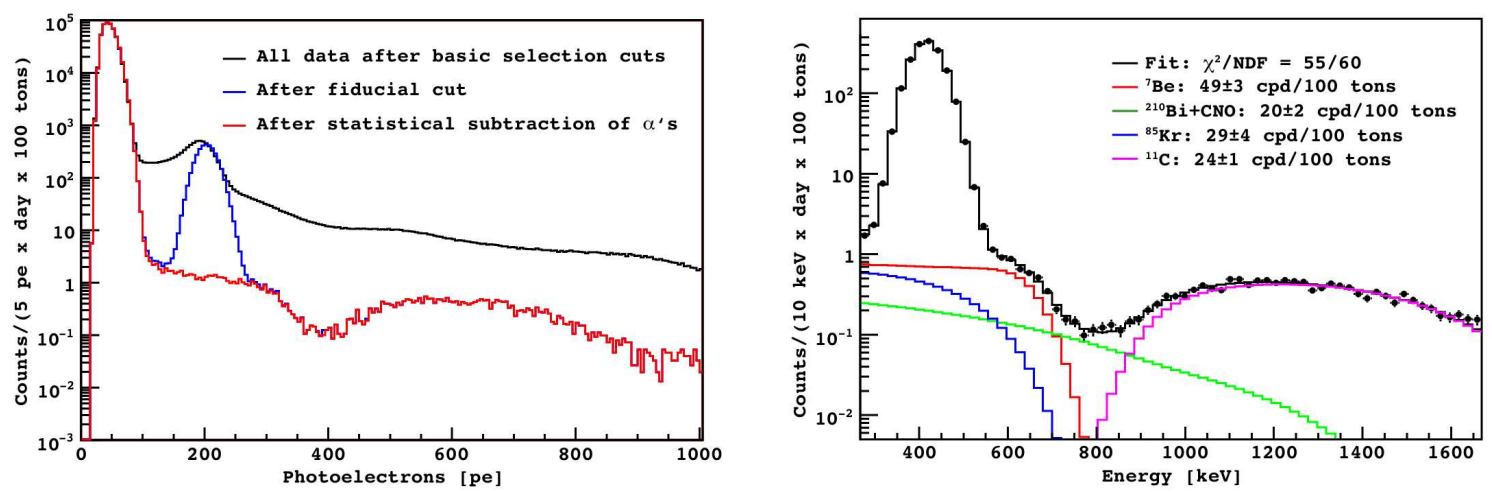

Figure 1: Left: The raw photoelectron charge spectrum after the basic cuts (black), after the fiducial cut (blue), and after the statistical subtraction of the $\alpha$-emitting contaminants (red). All curves scaled to the exposure of 100 day·ton. Right: Spectral fit in the energy region 260-1670 keV prior to statistical $\alpha$ ś subtraction.

of a set of concentric shells of increasing radiopurity and through an accurate selection of all the construction materials and operational procedures. Details can be found elsewhere [1].

\section{Radiopurity and Background levels}

The study of the time correlated events belonging to the ${ }^{238} \mathrm{U}$ and ${ }^{232} \mathrm{Th}$ radioactive chains yields, under the hypothesis of saecular equilibrium, an internal contamination for ${ }^{238} \mathrm{U}$ of $1.6 \pm 0.1$ $\cdot 10^{-17} \mathrm{~g} / \mathrm{g}$ and for ${ }^{232} \mathrm{Th}$ of $6.8 \pm 1.5 \cdot 10^{-18} \mathrm{~g} / \mathrm{g}$. The concentration of these contaminants is significantly lower than the design value of $10^{16} \mathrm{~g} / \mathrm{g}$ and it is not the main issue of the ${ }^{7} \mathrm{Be}$ analysis. On the contrary, the most important background is due to the $\beta$ decay of ${ }^{85} \mathrm{Kr}$ with $687 \mathrm{KeV}$ end point having a rate of the same order of magnitude of the ${ }^{7} \mathrm{Be}$ signal. The analysis of the rare decays of ${ }^{85} \mathrm{Kr}$ into ${ }^{85} \mathrm{Rb}$ (branching ration $0.43 \%$ but taggable due to the presence of time correlated events) yields $28 \pm 7$ counts/(days $\cdot 100 \cdot t)$ after a live time of more than 1 year. Additional background is identified as ${ }^{210} \mathrm{Bi}$ and as ${ }^{11} \mathrm{C}$. The last one is produced by the interaction of muons in the scintillator.

\section{Data analysis and results}

The curves presented in Fig. 11 (left) are the results of 192 live days of measurement. The black line demonstrates the raw data with the 3 most basic cuts already applied. This means, that only single clustered events are accepted in order to reject pile up or fast coincidences. Second all muons are neglected. This tagging can be performed using the OD as an active muon veto. The efficiency is $99.5 \%$. In addition, muons and neutrinos can be distinguished by pulse shape analysis using the ID. The remaining inefficiency is reduced to less than $10^{-3}$. And third, as a logical consequence, all events detected in a $2 \mathrm{~ms}$ time window after each muon, are rejected in order to avoid muon induced secondaries. The blue curve is obtained using a fiducial volume cut to reject external $\gamma$ background: The allowed detector volume corresponds to the innermost 100 tons 
( $\sim 3.25 \mathrm{~m}$ radius). Also the radon induced ${ }^{214} \mathrm{Bi}^{2}{ }^{214} \mathrm{Po}$ coincidences are removed from data. The large ${ }^{14} \mathrm{C}$ peak remains at the lower energy range of the observed spectrum, which therefore will not influence the results of the ${ }^{7} \mathrm{Be}$ neutrinos. Another remaining obvious part is the still present ${ }^{210} P o$ peak at about 190 pe. It can be rejected by $\alpha / \beta$ pulse shape discrimination (red curve). In both cases, blue and red line, the compton like edge of the ${ }^{7} \mathrm{Be}$ neutrinos (300-350 pe) and the ${ }^{11} \mathrm{C}$ spectrum (400-800 pe) becomes visible. In Fig. 1 (right), as final result, the fits applied to the remaining data are presented [⿰]⿻ 丨. Two independent methods are used for this evaluation and come to consistent results (expressed in counts/day/100tons, statistical errors only): $49 \pm 3$ for ${ }^{7} \mathrm{Be}, 20 \pm$ 2 for a sum of CNO-neutrinos and $210 \mathrm{Bi}, 29 \pm 4$ for ${ }^{85} \mathrm{Kr}, 24 \pm 1$ for ${ }^{11} \mathrm{C}$. With an estimation of the systematic error $(8.5 \%)$ a ${ }^{7} \mathrm{Be}$ neutrino rate of $(49 \pm 3$ (stat) \pm 4 (sys) ) counts/day/100tons can be announced. The rate expected without neutrino oscillation for the high metallicity Standard Solar Model (SSM) (BS07(GS98)) is (74 \pm 4 ) counts/day/100tons. The flux normalization constant $\mathrm{f}$, the ratio between the measured MSW-LMA scenario and the predicted BS07(GS(98)) neutrino flux, is calculated to $\mathrm{f}_{B e}=1.02 \pm 0.10$. The observed survival probability at the ${ }^{7} \mathrm{Be}$ energy of $862 \mathrm{keV}$ is $\mathrm{P}_{e e}=0.56 \pm 0.10$. The no oscillation hypothesis $\left(\mathrm{P}_{e e}=1\right)$ is rejected at $4 \sigma$ C.L.. So Borexino confirms the MSW-LMA neutrino oscillation scenario and provides the first direct measurement in the low-energy vacuum MSW regime. As result of this preliminary analysis, a ${ }^{8} \mathrm{~B}$ neutrino rate of $(0.26 \pm 0.04$ (stat) \pm 0.02 (sys) ) counts per day and 100 tons down to an energy of $2.8 \mathrm{MeV}$ can be reported [5]. The corresponding $v_{e}$ flux of $\left(2.65 \pm 0.44\right.$ (stat) \pm 0.18 (sys0 ) $\cdot 10^{6} \mathrm{~cm}^{-2} \mathrm{~s}^{-1}$ is in agreement with the MSW-LMA SSM prediction and SNO or Super-Kamiokande results. Assuming the BS07(GS98) SSM, the mean survival probability is $0.35 \pm 0.10$ at the effective energy of 8.6 $\mathrm{MeV}$. The no-oscillation scenario can be excluded using this preliminary analysis at a $4.2 \sigma$ C.L.. Borexino is the first experiment with the ablility to simultaneous measure solar neutrinos from the vacuum region $\left({ }^{7} \mathrm{Be}\right)$ and from the matter-enhanced oscillation region $\left({ }^{8} \mathrm{~B}\right)$. The ratio between the two different survival probabilities for ${ }^{7} \mathrm{Be}$ and ${ }^{8} \mathrm{~B}$ neutrinos is $1.60 \pm 0.33,1.8 \sigma$ different from unity. Still at relatively low significance, this result confirms the transition between low energy vacuum dominated and high energy matter enhanced solar neutrino oscillation, as predicted by the MSW-LMA solution.

\section{The future of Borexino}

Given the exceptional, unprecedented purity results achieved in Borexino, there are plenty of goals which we would like to achieve. A broad investigation of the solar $v$ spectrum is well within our reach. A calibration campaign has been performed and theanalysis of these data is in progress. In the near future, the systematic error can be substantially reduced. We aim at reducing the error of the ${ }^{7} \mathrm{Be}-v$ measurement down to $5 \%$. A feasibility of measurement of pep, CNO and possibly pp solar $v$ is under an extensive study. The detection of geo-neutrinos in Borexino is also in progress and we expect to collect a statistically significant sample of events in few years. Borexino has also strong potential in the field of supernovae (anti-)neutrinos. Borexino Collaboration joined the SNEWS project which involves an international collaboration of experimenters representing current supernova neutrino detectors. Detection of low energy neutrinos from the last stages of nuclear burning may help to foresee a massive star death a few days before its core collapse [6]. 


\section{Acknowledgements}

The Borexino program was made possible by funding from INFN (Italy), NSF (USA), BMBF, DFG and MPG (Germany), Rosnauka (Russia) and MNiSW (Poland).

\section{References}

[1] H. O. Back et al. (Borexino Collaboration), Nucl. Instrum. Methods Phys. Res., Sect. A 584, 98 (2008)

[2] C. Arpesella et al. (Borexino Collaboration) Phys. Lett. B 658101 (2008)

[3] A. Yu. Smirnov arXiv:hep-ph/0305106v1

[4] C. Arpesella et al. (Borexino Collaboration) Phys.Rev.Lett.101:091302,2008

[5] G. Bellini et al. (Borexino Collaboration), arXiv:0808.2868

[6] Odrzywolek, Misiaszek and Kutschera, Astropart. Phys. 21 (2004) 303-313 